\title{
De Cambeyrac à Strashourg: Quinze ans d'exploitation des groupes bulbes
}

\author{
par II. André \\ Ingénieur E.D.F. (Service de la Production Hydraulique)
}

Ont participé plus particulièrement à l'élaboration de ce document:

MM. CAZENAVE et DE CHABERT (C.N.R.) - M. CASACCI (Neyrpic)

MM. CAVAILLË et FRANCOIS (E.D.F., Etudes et Recherches) - MM. BARRES,

DOUILLET et LAVY (E.D.F., Production Hydraulique, D.T.G.) - MM. BORIES,

CABROL, CORNILLON, CORRE, DOTT, DUFAUT, MARCHAT et

SANDRET (E.D.F., Production Hydraulique, G.R.P.H.)

\section{Préambule}

Depuis le premier tour de roue, en 1957, du groupe 1 de Cambeyrac, 28 groupes bulbes de grandes dimensions et de puissances unitaires supérieures à $5 \mathrm{MW}$, auxquels il faudrait ajouter les 24 bulbes de la Rance, ont été mis en service sur les rivières françaises, les derniers aménagements en date étant Saint-Vallier (Gervans) sur le Rhône et Strasbourg sur le Rhin.

Ce sont sur les performances et sur les résultats d'exploitation de ces 28 groupes et de leurs modèles réduits, plus que sur les bulbes de faible puissance, que se sont penchés, tout particulièrement, les nombreux ingénieurs de la Compagnie Nationale du Rhône, de Neyrpic et 'de l'Electricité de France, qui ont coopéré à la réalisation de cette communication.

\section{Petits bulbes.}

L'expérience des petits groupes bulbes montre qu'ils ont, généralement, bien rempli leur contrat dans le cadre de très faibles chutes et de débits módestes, l'utilisation la plus courante étant l'injection directe de courant à basse tension dans les réseaux locaux de distribution, une autre des utilisations appréciées concernant les groupes de restitution placés dans les barrages de prises d'eau.

M. Cotillon fait, dans sa communication, lanalyse des diverses techniques utilisées et des résultats auxquels elles ont conduit; quelques conclusions générales s'imposent plus particulièrement pour l'exploitant :
Dans le domaine mécanique, les paliers lisses sont plus robustes que les roulements à billes ou à rouleaux, mais ceux-ci donnent toute satisfaction 'dans la mesure où ils sont très largement dimensionnés, particulièrement pour les butées.

Certains ennuis sérieux ont été provoqués par les joints d'arbres. Ceux-ci demandent des montages très soignés et donnent souvent toute satisfaction (Basse-Maulde). Toutefois, ce problème n'est pas spécifique des bulbes, même s'il revêt une acuité plus grande pour ce type de machines.

Pour les alternateurs, les groupes dans l'air ont une supériorité manifeste sur les groupes dans l'huile. Les problèmes d'usure de balais et de poussières de charbon seront évoqués à propos des groupes bulbes de forte puissance.

\section{Gros bulbes.}

En ce qui concerne ces derniers, leurs performances et les résultats d'exploitation, la constatation qui s'impose est que les exigences des hydrauliciens posent des problèmes aux électriciens, conduits, en raison du faible diamètre des alternateurs, à demander beaucoup à leurs machines : peutêtre faut-il voir une revanche inconsciente des hydrauliciens souvent contraints, par leurs collègues des Ponts, à considérer que les routes doivent passer sur les rivières plutôt que les rivières sous les ponts.

En règle générale, les problèmes liés à l'hydraulique sont souvent mieux résolus pour les bulbes que pour les Kaplan, mais la construction des alternateurs exige certaines précautions particulières et les mécaniciens ont à assembler et fixer de lourdes pièces horizontales en porte-à-faux. 


\section{1. - Contrôle des performances}

\subsection{Essais électriques.}

\subsubsection{RENDEMENTS DES ALTERNATEURS.}

Les pertes sont, proportionnellement, un peu plus élevées pour les alternateurs de groupes bulbes que pour les machines traditionnelles de même puissance. Ce résultat est lié essentiellement aux faibles dimensions radiales, imposées par l'hydraulique, conduisant à des entrefers très petits et à des flux importants. Les pertes mesurées sont comprises entre $2,80 \%$ et $3,80 \%$ de la puissance pour un $\cos \varphi$ voisin de 0,98 , valeur généralement admise pour les gros groupes bulbes.

\section{Méthode de mesures des pertes.}

Les méthodes traditionnelles ne sont pas applicables ou exigent des précautions particulières.

Pertes fer. - La méthode du moteur asynchrone, roue noyée et vannage légèrement ouvert, peut s'appliquer dans la mesure où tous les paramètres hydrauliques restent constants, ce qui n'est pas toujours facile à réaliser.

La méthode calorimétrique, avec tarage du circuit d'air à l'arrêt, semble possible, mais elle est contestée par certains, dans le cas des bulbes.

Pertes supplémentaires. - La méthode des ralentissements est difficile à appliquer dans le cas des bulbes, et est également contestée.

La méthode par tarage de l'échauffement de l'air, à partir des pertes fer mesurées en moteur synchrone, a l'inconvénient de nécessiter une extrapolation de la courbe de tarage (pertes supplémentaires importantes).

La méthode par tarage de l'échauffement de l'air à l'arrêt suppose que les débits d'air ne sont pas modifiés entre l'arrêt et la rotation. Elle nécessite la mise en jeu d'une puissance en courant continu très importante.

Pertes par ventilation propre (brassage). - La valeur mesurée dépend beaucoup de la position des capteurs thermiques.

Pertes d'excitation. - Elles varient dans de grandes proportions, près de $15 \%$ avec la température (variations de l'entrefer avec la température). Les normes U.T.E. ne prévoyant pas la température de mesures des caractéristiques, l'interpolation des courants à partir des points en charge, stabilisés en température est la seule mesure sérieuse.

\subsubsection{ECHAUfFEMENTS.}

Les échauffements sont souvent très inférieurs aux limites contractuelles, aussi bien pour les enroulements statoriques que rotoriques. Il s'agit toutefois des échauffements moyens, mais il serait intéressant de pouvoir connaitre les valeurs locales des températures, car on peut redouter de forts gradients dans les rotors de groupes bulbes.

Sur les groupes où les valeurs étaient proches des garanties, c'était, en général, à cause de l'encrassement des surfaces réfrigérantes ou de l'emploi de peintures particulièrement isolantes pour la carcasse (voir paragraphe 2.1).

\subsubsection{Caractéristiques transitoires.}

Les groupes bulbes sont maintenant excités par diodes tournantes, ce qui complique les mesures de certaines caractéristiques par suite de l'impossibilité d'accéder directement aux grandeurs électriques du rotor par l'intermédiaire des bagues et balais.

Ce problème, commun à tous les alternateurs ayant ce mode d'excitation, peut être résolu, soit en transmettant par radio le courant et la tension d'excitation, soit en posant pour les mesures trois bagues et des balais.

On peut résoudre ce problème en adoptant certaines méthodes de mesures groupe à l'arrêt, mais ces méthodes ne sont pas encore toutes reconnues par les organismes de normalisation.

\subsection{Mesures hydrauliques - Conditions d'écoulement.}

\subsubsection{RENDEMENTS.}

Les rendements mesurés sur les bulbes sont bons: $88 \%$ à $92,5 \%$ et soutiennent la comparaison avec ceux des meilleures turbines Kaplan.

Les débits spécifiques des bulbes sont très supérieurs à ceux des Kaplan (de même diamètre et de même vitesse).

Les groupes bulbes ont fait l'objet de nombreux essais sur modèles réduits, mais le nombre de mesures effectuées dans de bonnes conditions et permettant de déterminer des valeurs absolues du rendement sur les machines in'dustrielles est assez réduit. On peut citer les cas de Cambeyrac, Saint-Malo, la Rance, Pierre-Bénite, Gerstheim, BeaumontMonteux et Le Prat. Il n'apparait aucun écart systématique entre les valeurs mesurées et les valeurs prévues d'après les essais sur modèles.

On peut citer:

a) Le cas de la Rance, où les machines industrielles ont révélé un meilleur rendement que celui prévu par le modèle pour la marche en turbine «directe», un déficit dû à la cavitation en turbine inverse pour les chutes supérieures à $5 \mathrm{~m}$, et le rendement prévu pour les fonctionnements en pompe dans les deux sens.

b) Le cas de Gerstheim, où la comparaison a pu être faite pour un des types de groupes bulbes et sous la chute de $12 \mathrm{~m}$ : le rendement maximal a été trouvé pour le débit prévu, le rendement de la machine industrielle est supérieur de deux points environ au rendement déduit des essais sur modèle, mais les tolérances de mesure doivent être du même ordre de gran'deur.

\subsubsection{CAMES DE CONJugaison.}

Les contrôles en fonctionnement des machines in'dustrielles ont fait apparaître des écarts faibles (sauf dans un cas) entre les valeurs optimales de rendement et les rendements obtenus à partir de la came fournie par le constructeur et (en principe) $\left({ }^{a}\right)$ déduite des essais sur modèles. Il faut remarquer que la méthode d'optimisation, qui consiste à rechercher la valeur minimale des pertes dans l'aspirateur

(i) Il est en pratique difficile de vérifier que les cames en service sont identiques à celles déduites du modèle, ne serait-ce parce que les paramètres utilisés pour l'usinage ne sont pas toujours les mêmes (ouverture en degrés ou en 1/10). 
(mesurées au tube de Pitot), conduit sensiblement aux mêmes résultats que la méthode consistant à minimiser les pertes globales (contrôle piézométrique), ceci étant vrai pour les bulbes comme pour les Kaplan. Au niveau des méthodes de mesures, les contrôles piézométriques peuvent être utilisés, mais ils doivent l'être avec discernement, en choisissant avec soin les emplacements des prises de pression : on considère généralement, en 1972, que l'on a intérêt à placer les prises le plus loin possible à l'aval et non sur les bras supports amont comme on l'avait envisagé initialement; ces emplacements, mal choisis, conduisaient à des instabilités de plus de $5 \%$ dans les pressions différentielles.

Il faut souligner que l'optimisation des conjugaisons, d'une part entraîne des gains d'énergie, qui sont importants même lorsque les écarts entre la conjugaison optimale et la conjugaison initiale sont faibles $(1 \%=5$ à 20 millions de $\mathrm{kWh}$ par an sur chacune des usines du Rhône ou du Rhin), et d'autre part diminue la cavitation, donc les frais d'entretien et les pertes d'énergie.

Une recherche a été effectuée par la C.N.R. en vue de déterminer un paramètre sensible au réglage de la conjugaison. A Gervans, au cours du fonctionnement en hélice bloquée (au voisinage de la chute maximale), on a remarqué que le spectre des vibrations au droit des pales (capteur placé sur le manteau), dans la plage de 20 à $50 \mathrm{~Hz}$, était très sensible à la surouverture du vannage, ainsi qu'il ressort de la comparaison des figures 1 (conjugaison correcte) et 2 (vannage en surouverture). Le dispositif de mesure en question a permis d'expérimenter, sur les groupes de Gervans, les conditions de fonctionnement des groupes de Sauveterre (à pales et vannage fixe), lors du démarrage et de l'arrêt réalisé par la vanne aval.

Les études sur modèles réduits, faites pour Cambeyrac, ont donné d'excellents résultats pour la détermination des pertes de l'aspirateur, les coefficients de récupération et de répartition des vitesses; les résultats obtenus pour la Rance sont un peu moins satisfaisants, en particulier pour la cavitation.

\subsubsection{Localisation des pertes.}

Les essais industriels ont montré que le rendement des aspirateurs de bulbes était très bon et comparable à celui des meilleures turbines Kaplan, mais qu'il n'était pas meilleur, contrairement à ce qu'on attendait. L'amélioration conduirait d'ailleurs à des gains minimes. Les pertes, en amont de la roue, sont, au contraire, plus faibles pour les bulbes, en raison des différences d'écoulements qui comportent de plus forts gradients de vitesse angulaire pour les Kaplan que pour les bulbes, ces gradients étant source de pertes.

Les pertes les plus importantes proviennent de la roue et il est admis, à la suite de la réalisation de la roue expérimentale de Beaumont-Monteux, que le tracé d'un bulbe est spécifique, différent de celui d'une turbine Kaplan.

\subsubsection{RÉGIMES TRANSITOIRES.}

En raison du caractère axisymétrique de l'écoulement dans une turbine de groupe bulbe, tous les filets liquides ont la même longueur minimale $L$, ce qui conduit à des temps caractéristiques $(\theta=\mathrm{LV} / g \mathrm{H}$, où $\mathrm{V}=$ vitesse et $\mathrm{H}=$ chute) du coup de bélier de masse nettement plus faibles $(50 \%)$ que pour les Kaplan, et à des fonctionnements plus stables en régime transitoire.

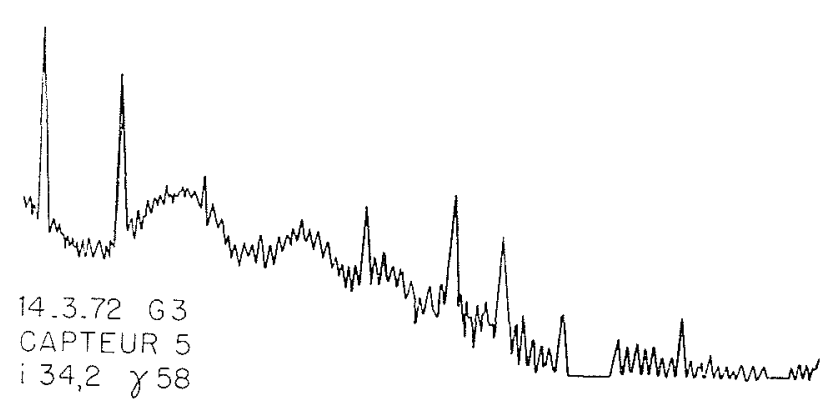

1/ Vibrations du manteau de roue. Capteur placé au droit des pales / a) Conjugaison correcte.

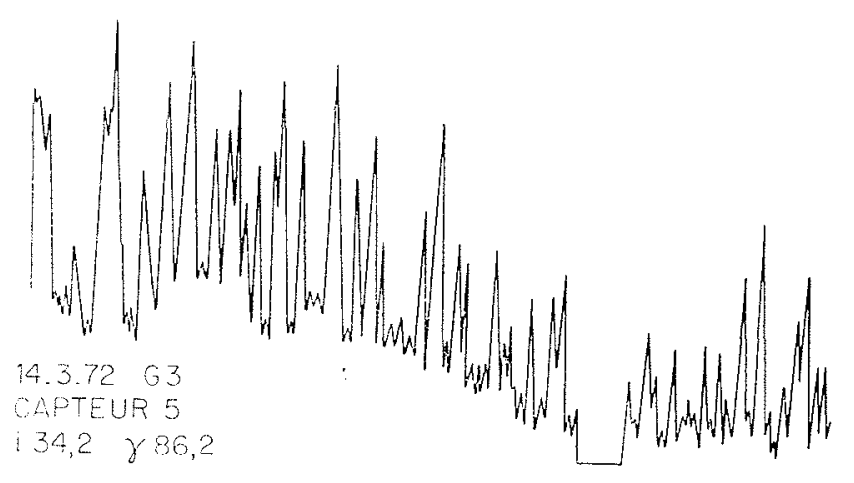

2/ Vibrations du manteau de roue. Capteur placé au droit des pales ( b) Conjugaison incorrecte (surouverture du vannage).

Les retours en marche à vide, après déclenchement à pleine charge, sont accompagnés d'une oscillation du vanrage et de la vitesse. Comme sur les Kaplan, le régime le plus sévère a lieu au cours de la décélération après emballement (fluctuation des vitesses, dissymétries, d'où flexion d'arbres, inversion de la poussée hydraulique). Le déclenchement, avec passage en marche en déchargeur, a des effets analogues à ceux d'un déclenchement simple, moins sèvère dans le cas du réglage du débit par vanne aval.

\subsubsection{Marche EN DÉChargeUr.}

Les essais effectués à Beaumont-Monteux, sur le Rhin et sur le Rhône, n'ont mis en évidence aucune particularité très remarquable; il semble que t'on puisse accepter une déconjugaison plus importante sur les bulbes que sur les Kaplan.

La marche en déchargeur permet de réduire considérablement l'amplitude de l'onde de coupure: ainsi, à Bealtcaire, pour un débit coupé de $2000 \mathrm{~m}^{3} / \mathrm{s}$, l'amplitude est ramenée de $1,25 \mathrm{~m}$ à $0,27 \mathrm{~m}$ au droit 'de l'entrée 'd'eau. Les essais de coupure, faits sur les groupes verticaux et sur les bulbes, ont montré que l'onde principale est analogue, un peu plus forte sur les bulbes, mais que les ondes suivantes sont nettement plus faibles sur les bulbes. Il faudrait, toutefois, étudier plus en détail la vitesse de fermeture comparée dans le cas des bulbes et des Kaplan. 
a) Débit contrôlé par vanne aval: Le régime est stable, quel que soit le type de groupe.

b) Débit contrôlé par le vannage de la turbine: Le débit déchargé varie entre $35 \%$ et $75 \%$ du débit nominal, pour des ouvertures de vannage comprises entre $2 / 10$ et $6 / 10$; l'influence de l'ouverture de la roue (pour les grandes ouvertures) est relativement faible, bien que ces ouvertures aient une influence sur le pourcentage d'énergie dissipée à l'aval de la roue et qui peut atteindre $50 \%$ de l'énergie totale.

La vitesse de rotation varje en fonction inverse de l'ouverture des pales.

Les efforts de manœuvre sur le vannage sont accrus, du fait que le débit est plus grand dans la marche en déchargeur qu'en marche normale, pour une ouverture donnée.

Les sollicitations du groupe sont plus fortes qu'en marche normale (poussée hydraulique, pulsations de vitesse, flexion alternée de l'arbre) et d'autant plus élevées que le débit est plus élevé.

On a été amené à limiter la marche en déchargeur à une durée d'une demi-heure dans certains cas.

\subsubsection{ESSAIS SPÉCIAUX.}

\section{a) Sur modèles réduits (La Rance):}

Vannes de coupure aval. - Les premiers essais sur modèle ont fait apparaître des excédents d'efforts verticaux conduisant à des risques certains: les améliorations apportées sur les vannes industrielles ont donné des résultats satisfaisants.

Démarrage des groupes. - Des anomalies ayant été constatées à la Rance dans les opérations de démarrage, pour les chutes supérieures à $6 \mathrm{~m}$, ces fonctionnements ont été reproduits sur modèles, ce qui a permis de donner les indications nécessaires au démarrage et à l'arrêt pour toutes les situations de chute.

\section{b) Sur machines industrielles:}

Des essais, portant sur le comportement des pales lors des coupures en charge, vont être effectués en plaçant des jauges de contrainte sur une pale de la roue, la liaison avec l'enregistreur se faisant par radio.

Dans le domaine particulier de l'alternateur, des essais sont entrepris en vue de suivre le comportement du circuit magnétique lors de la montée en température de l'alternateur (démarrage rapide).

On peut également signaler que l'installation d'une caméra de télévision, dans le compartiment palier de la turbine (inaccessible en marche), permet de surveiller, pendant les premiers tours de roue (marche mécanique), les comparateurs placés sur l'arbre et les thermomètres et manomètres de pression d'huile placés sur le palier.

\section{2. - Résultats d'exploitation}

On peut considérer que, dans l'ensemble, les résultats d'exploitation sont satisfaisants, après les mises au point de la première année succédant à la mise en service.

Les valeurs figurant dans le tableau 1 illustrent bien la qualité des services rendus.

\begin{tabular}{|c|c|c|}
\hline & & Tableau 1 \\
\hline \multirow{2}{*}{ USinES } & \multicolumn{2}{|r|}{ Production anNuELle } \\
\hline & $\begin{array}{l}\text { prévue } \\
\text { (GWh) }\end{array}$ & $\begin{array}{l}\text { réelle } \\
\text { (GWh) }\end{array}$ \\
\hline Cambeyrac & 27 & moyenne 1960-1971: 35 \\
\hline Argentat $\ldots$ & 102 & moyenne 1959-1971: 112 \\
\hline Pierre-Bénite & 510 & $\begin{array}{ll}\text { en 1970: } & 553 \\
\text { en 1971: } & 443(*)\end{array}$ \\
\hline Gerstheim & 708 & moyenne 1968-1971: 733 \\
\hline Beaucaire & $\begin{array}{c}1020 \\
\left(1^{\mathrm{Hr}} \text { stade }\right)\end{array}$ & $938(*)$ \\
\hline \multicolumn{3}{|c|}{$\begin{array}{l}\text { Remarques: } \\
(*) \text { Faible hydraulicité, productibilité de l'ordre de } 0,85 \text {. }\end{array}$} \\
\hline
\end{tabular}

Parmi les incidents que l'on a pu constater, certains sont communs à tous les types de machines et d'autres peuvent être considérés comme spécifiques des bulbes. Ces derniers ont été résolus, soit par l'adoption d'une technologie particulière, soit par l'amélioration de la qualité des matériaux. et du montage.

Ils étaient liés essentiellement:

- pour l'altèrnateur, à l'exiguïté de la machine, aux flux et aux gradients thermiques importants;

- pour la turbine, à la mise en porte-à-faux de pièces lourdes en position horizontale.

Certains défauts, qui ne sont pas spécifiques des bulbes, y revêtent une acuité particulière; ils sont, le plus souvent, liés aux problèmes 'd'étanchéité.

\subsection{Alternateurs.}

\section{STATORS. - ISOLATION.}

En raison de la tension relativement basse, les problèmes d'isolation ne sont pas cruciaux. Toutefois, le calage des barres d'encoches doit être très soigné (montage sans jeu) pour que les intensités de courant par encoche, généralement élevées, n'entraînent pas d'altération de l'isolation par électro-érosion et martèlement (voir rotors).

Les essais ont montré que les isolations étaient, dans certains cas, assez basses après plusieurs jours d'arrêt et qu'il fallait sécher les groupes avant redémarrage, mais ceci ne semble pas s'être produit en exploitation courante.

Le fait qu'il soit nécessaire de traverser l'alternateur, pour accéder au moulinet, a conduit à des défauts sur les bobinages, par blessures de l'isolant lors des manipulations de matériel.

Toutes les solutions permettant l'accès direct à l'aval des groupes, sans traverser l'alternateur, sont donc intéressantes. Quand il est nécessaire de le traverser, il est utile de prévoir des caches pour protéger les enroulements. 


\section{CrRCUIT Magnétique.}

Un incident est à signaler sur un bobinage de stator par suite du mouvement de doigts de serrage d'un circuit magnétique; la solution, généralement a'doptée maintenant, prévoit un serrage des tôles du circuit magnétique par des tiges indépendantes des clavettes de guidage et réalisées en acier magnétique, isolées quan'd elles sont à l'intérieur du circuit magnétique, et semble donner toute satisfaction lorsque les doigts de serrage sont rigides.

La pression de serrage du circuit de 10 à 15 bars est satisfaisante; il semble que l'on puisse abandonner le collage des tôles du circuit magnétique, adopté par prudence à la Rance.

L'emploi de cales magnétiques, maintenant mises au point par certains constructeurs, mériterait d'être examiné pour diminuer les pertes et les taux d'harmonique.

\section{RoTOR.}

La plupart des incidents électriques constatés jusque-là proviennent du rotor.

\section{a) Electro-Érosion :}

Des césordres importants ont été constatés sur des pôles, après des durées de service comprises entre 5000 et $10000 \mathrm{~h}$, par suite de l'extrusion de barres d'amortisseurs de leur logement dans les pôles et de leur mise en contact avec le stator (très faible entrefer): ces incidents sont dus à un amorçage électrique entre la barre et les tôles, cet amorçage constituant la fermeture du circuit barre d'amortisseur-tôlesgoujons de serrage (voir fig. 3 ). Il en résulte un maigrissement des barres et une ouverture des fentes par suite des fortes tensions induites aux fréquences harmoniques dans les barres d'amortisseurs.

Une première solution consiste à isoler les barres d'amortisseurs, mais la solution est coûteuse. On peut, toutefois, se demander si la solution imprégnation des pôles sous vide et pression ne serait pas, finalement, la plus rationnelle. On peut également envisager l'emploi de pôles massifs sans barres d'amortisseurs.

Les remèdes réellement adoptés sont les suivants :

- réduction des tolérances sur le diamètre des barres et les diamètres d'alésage de leur logement;

- fermeture des logements 'de ces barres;

- trois points de soudure en bouchon, sous argon, de la barre sur la tôle, matage par point des isthmes;

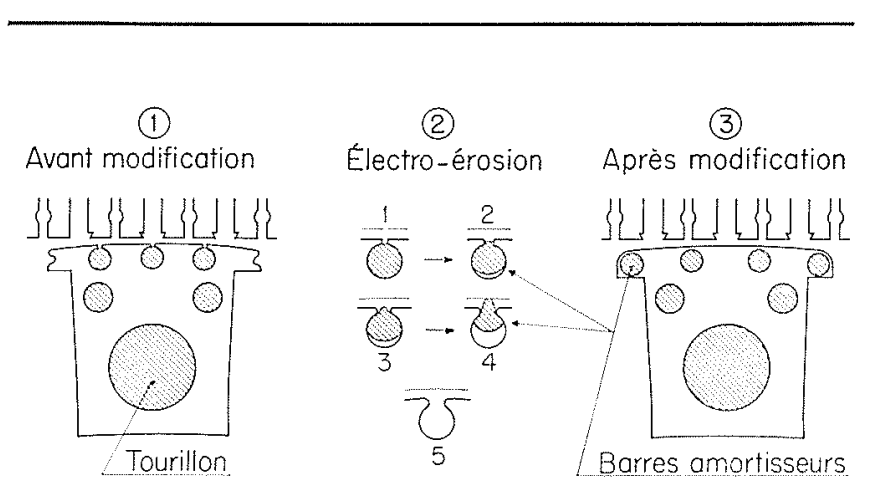

3/ Phénomène d'électro-érosion.
- quand on refait le pôle, modification de la répartition des barres avec, dans certains cas, suppression de l'une d'entre elles, pour diminuer les forces électromotrices de mutuelle inductance entre stator et amortisseur à la fréquence des harmoniques de denture, isolement des tôles par un vernis et légère augmentation de l'entrefer.

Ce type d'incident a été plus fréquent sur les bulbes, mais il avait été constaté ailleurs; il ne se produit plus sur les derniers groupes bulbes mis en service.

\section{b) BAISSE D'ISOLEMENT :}

Poussière de charbon. - On a fréquemment constaté une baisse inquiétante de l'isolement dans le cas où les dispositifs d'excitation utilisent des bagues et des balais à l'intérieur des enceintes des groupes: la poussière de charbon, provenant de l'usure souvent anormale 'des balais et véhiculée par la ventilation dans l'enceinte pressurisée, diminue l'isolement en se déposant sur les enroulements (parfois avec des vapeurs d'huile). Ces incidents sont spécifiques des bulbes.

Plusieurs remèdes peuvent être apportés :

- le dispositif le plus rationnel, qui est dorénavant adopté pour les nouveaux bulbes, est l'adoption d'une excitation par diodes tournantes;

- adoption de systèmes d'excitation dont les bagues et balais sont à l'extérieur de l'enceinte des groupes;

- quand il est nécessaire de conserver les bagues et balais à l'intérieur, on peut réduire l'usure des balais en adoptant des matériaux mieux adaptés.

Une méthode intéressante d'investigation, pour déterminer la nature des balais à adopter, consiste à prélever les gaz dans l'enceinte pressurisée et à les analyser par chromatographie. L'usure anormale des charbons provient, parfois, de la présence de gaz issus de la polymérisation des isolants. Dans l'ensemble, les balais à haute teneur en argent semblent donner de meilleurs résultats, surtout lorsqu'on augmente l'humidité de l'air ambiant. La surface des filtres du circuit de ventilation doit être largement dimensionnée, il est intéressant de prévoir un extracteur de vapeurs d'huile.

Humidité. - Les isolements du rotor sont très variables lorsque les groupes sont ouverts et peuvent baisser jusqu'à $20 \mathrm{k} \Omega$ lors de l'essai à $500 \mathrm{~V}$; il est facile de procéder au séchage par une soufflante $(24 \mathrm{~kW}, 24 \mathrm{~h}$ à Strasbourg).

Il est inutile de prévoir des déshumidificateurs à l'intérieur du bulbe, en raison de l'existence de la paroi froide et de l'impossibilité de condensation sur les parties fragiles par suite de leur température. Le cas de la Rance est particulier, car ces machines fonctionnent pendant plusieurs heures consécutives chaque jour «en orifice» et, durant ces périodes, on peut redouter le dépôt 'd'humidité en des points critiques.

Par ailleurs, dans les groupes pressurisés, l'air est partiellement déshydraté par suite de la compression $(5 \%$ à Gerstheim).

Quelles que soient les causes annexes des baisses d'isolement, il est apparu nécessaire de renforcer les isolations par projection de résine époxy sur les extrémités de bobine et de modifier les modes de connexion entre pôles, lorsqu'ils sont initialement montés pôles nord en série, pôles sud en série, afin d'adopter le montage «pôles nord et sud en série », plus difficile à placer dans les bulbes: c'est, en effet, le mode d'enroulement des pôles qui a été à l'origine de la gravité de l'accident dû à la poussière de charbon, un 
amorçage entre deux pôles pouvant mettre hors circuit toute une série de pôles.

L'utilisation des matériaux élastomères mal adaptés, sous les épanouissements polaires, a été la source d'autres ennuis; il semble que la simplicité du dispositif de fixation des pôles, par boulons sur la jante, donne satisfaction : des cales doivent être prévues, à l'origine, pour le réglage de l'entrefer.

On peut signaler un défaut assez particulier, concernant l'apparition de corrosion entre la jante du rotor et son croisillon sur lequel elle est montée, avec un léger serrage diamétral, et n'est maintenue que tangentiellement.

Carcasse. - Un point important est à souligner : le remplacement de peintures particulièrement isolantes, sur la paroi extérieure de la carcasse, par de la métallisation, a permis de gagner près de 20 degrés d'échauffement dans certains cas.

Pressurisation et réfrigérants. - La pressurisation a pour but essentiel de favoriser les échanges thermiques dont le coefficient est directement proportionnel à la masse spécifique de l'air. Dans les alternateurs conçus avec pressurisation, il est souvent possible de monter le groupe en puissance, en l'absence de pressurisation, jusqu'aux deux tiers de sa puissance nominale sans échauffement excessif.

Il semble possible, en adoptant des ailettes ou des tubes sur la carcasse, d'attein'dre des valeurs plus grandes de la puissance sans pressurisation. Toutefois, il est indispensable d'étudier de très près le problème des prix de revient, avec et sans pressurisation, avant de prendre une décision.

Régulation de tension. - Dispositifs d'excitation. Réactif. - En raison des incidents relatifs aux poussières de charbon lorsque les dispositifs d'excitation comportent 'des bagues et balais à l'intérieur de l'enceinte et, de façon plus générale, par suite de l'évolution des techniqques, le système d'excitation retenu le plus fréquemment est à diodes tournantes: celles-ci donnent toute satisfaction, dans la mesure où on prend les précautions nécessaires pour protéger le système contre les surtensions inverses qui peuvent prendre naissance, en particulier lors des couplages qui se font généralement en asynchrone. On n'a jamais constaté d'incidents lorsque ces diodes sont largement dimensionnées (cas général des diodes supportant une tension inverse de $2400 \mathrm{~V})$.

Les constructeurs ont conçu des dispositifs de protection des diodes contre les surtensions, soit par des éléments écrêteurs, par exemple pont de thyristors contrôlé par diodes Zener, qui ferme, en cas d'élévation de tension, un circuit de 'décharge résistif, soit par des fusibles rapides; d'autres sont constitués par des résistances VDR à caractéristiques variables en fonction de la tension. Il est nécessaire de disposer d'un dispositif sûr de détection des claquages des diodes ou fusibles pour tous ces systèmes d'excitation à redresseurs tournants.

Il est par ailleurs difficile de détecter les «masses rotor» sur les machines à excitation statique.

Un dispositif, qui semble donner toute satisfaction depuis deux ans, après une laborieuse mise au point, consiste à surveiller l'isolement global de l'ensemble: rotor de l'excitateur + diodes tournantes + bobines polaires du rotor et à transmettre, par induction mutuelle entre deux bobines, l'une fixe l'autre mobile, un signal lorsque la valeur de l'isolement atteint un seuil (voisin de $10 \mathrm{k} \Omega$ par exemple).

Les possibilités de fourniture ou d'absorption de réactif par des alternateurs des groupes bulbes sont faibles, mais elles ne sont pas entièrement négligeables comme on a tendance à le dire : -10 à $+10 \mathrm{MVAR}$ pour des puissances actives de $20 \mathrm{MW}$ par exemple (voir fig. 4).

\subsection{Turbines.}

Les soucis qu'ont donnés aux exploitants, dans ce domaine, les groupes bulbes, concernent essentiellement les usures par cavitation, les problèmes de joints et les assemblages boulonnés.

\section{Cavitation:}

Comme pour toutes les turbines, les paramètres liés à la cavitation sont nombreux et il est difficile de déterminer la prépondérance de chacun d'eux, la synthèse de toutes les constatations peut ceperidant être présentée de la façon suivante :

1) Après plus de 20000 heures de fonctionnement, on n'a pas constaté de traces de cavitation sensibles lorsque les pales des roues et le manteau sont en acier fortement allié $(17 / 4,13 \%$ de chrome ou 18/8).

2) Le phénomène de cavitation semble, sur les bulbes, plus influencé que sur les Kaplan par l'enfoncement de la machine; ceci paraît plausible, surtout pour les grands bulbes dont le diamètre est voisin de la moitié de la chute $6 \mathrm{~m}$ pour $12 \mathrm{~m}$ par exemple): on constate alors une cavitation exceptionnelle sur les demi-manteaux supérieurs et les essais sur modèles ont permis de constater que les décollements n'étaient pas les mêmes, pour les pales, lorsqu'elles passaient près de la génératrice inférieure ou près de la génératrice supérieure du manteau. Le calage, fait en fonction du point haut, est donc plus sévère que le calage fait pour les Kaplan au point moyen.

On signale un cas de cavitation sur le seul demi-manteau inférieur.

3) La cavitation est intimement liée à la mauvaise conjugaison pales-vannage, particulièrement lorsque le tracé des pales conduit à des bords d'attaque minces et «aigus», phénomène bien connu des aérodynamiciens.

4) Les constatations, faites sur des pales en acier ordinaire ou faiblement allié, montrent que la cavitation apparaît d'abord, en général, sur l'extrados des pales, près du bord d'attaque, puis, à un degré moindre, sur la face d'intrados du bord d'attaque. Dans certains cas, est apparue une cavitation marginale se développant le long du bord circonférentiel d'extrados et de la tranche.

Après 40000 heures, les zones cavitées ont atteint, dans les cas les plus graves, une bande de $500 \mathrm{~mm}$ sur toute la largeur de la pale, depuis le bord d'entrée, et une bande de $100 \mathrm{~mm}$ de largeur sur une grande longueur circonférentielle (fig. 5).

En l'absence de matériaux inoxydables, et sur un aménagement en service donné, lcs remèdes apportés sont essentiellement l'amélioration de la conjugaison, la limitation de l'ouverture et des retouches de profil.

Des études, entreprises par Neyrpic, la C.N.R. et E.D.F., ont apporté récemment de précieuses indications sur les relations entre les divers types de marche de la turbine et les différentes formes et emplacements de la cavitation. On trouvera ces renseignements dans l'exposé de M. Casacci. 
JoINTS :

Les problèmes sont les mêmes que pour les turbines traditionnelles, mais les conséquences des fuites sont souvent plus grandes, ce qui a conduit les exploitants à y prêter une attention plus particulière.

Joints d'arbres. - D'est en ouest, du sud au nord de la France, les mêmes joints d'arbres ne posent aucun problème ou font enrager leurs utilisateurs: comme pour la cavitation, et malgré le grand nombre d'essais enregistrés sur toutes sortes de turbines, il est difficile de tirer des conclusions définitives, toutefois:

1) L'usure 'des joints et l'augmentation des fuites sont essentiellement liées, quel que soit le type de joints :

a) à la qualité du montage;

b) à la qualité et à la nature des matériaux en suspension dans l'eau turbinée ou dans l'eau de refroidissement des joints;

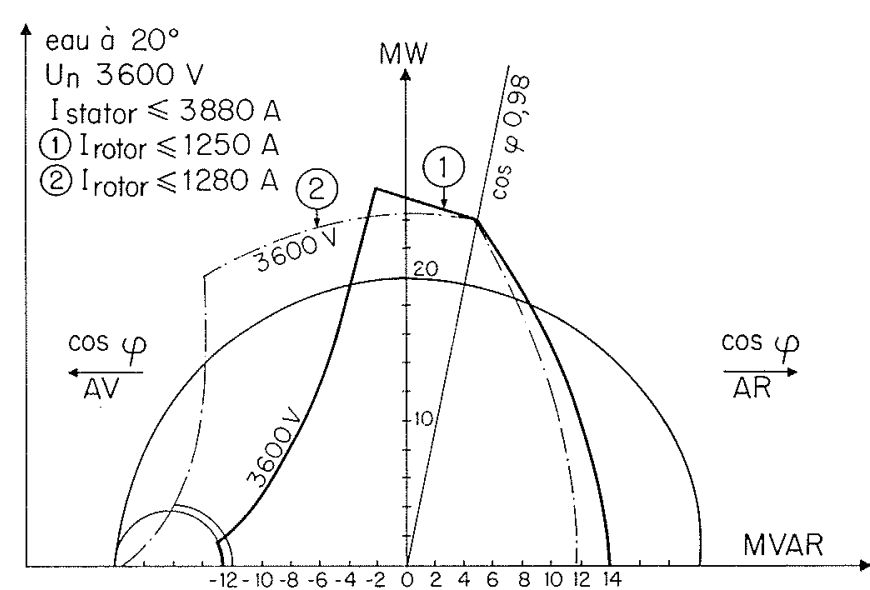

4/ Diagrammes stabilité. Puissance active et réactive de deux gros bulbes $24000 \mathrm{kVA}$.

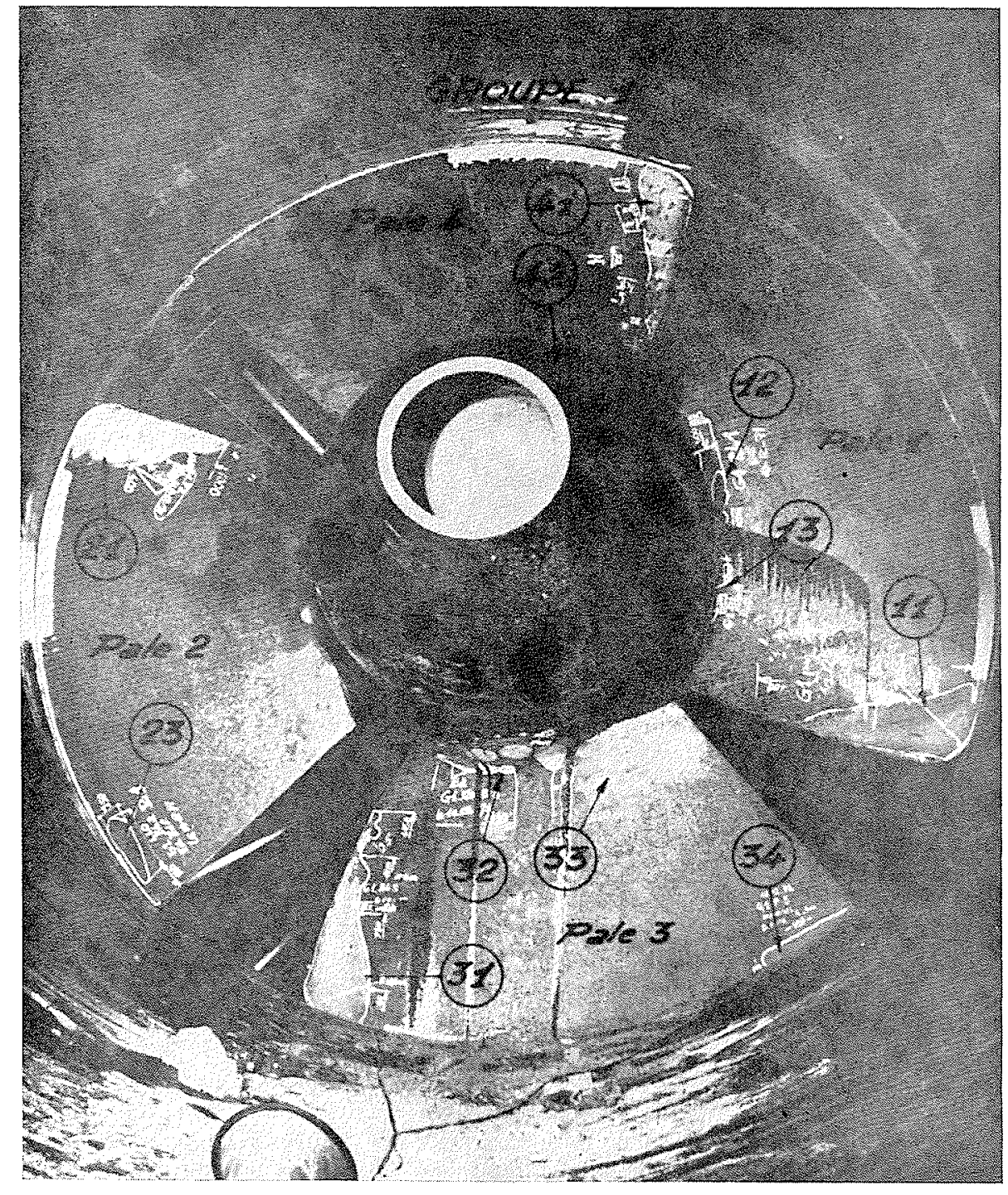

5/ G. 1. - Ensemble des pales. Extrados. Visite du 10-12-1971. 
c) à la vitesse linéaire (notion du nombre de kilomètres parcourus en un an);

d) au phénomène hydrodynamique de formation du film d'eau qui exige, dans tous les cas, que l'on apporte un grand soin au montage, que l'on étudie la forme du joint et que l'on fournisse l'eau d'arrosage des joints, même à l'arrêt.

2) Les joints plans supportent mal les variations de pression de marche transitoire. Toutefois, un nombre croissant d'exploitants semblent préférer les joints plans aux joints radiaux.

3) Pour les joints cylindriques, dans certains cas, les joints au carbone ont donné satisfaction, dans d'autres cas, il semble préférable de les remplacer par des matériaux «type polyamide, polyéthylène ou nylon ».

On a quelquefois amélioré les résultats en réalisant des chambrages, pour amener la pression sur la face d'appui du joint, et réduire ainsi la pression spécifique, en particulier, sur la quatrième rangée des joints plans la plus sollicitée (amorçage du film d'eau).

4) Les douilles en bronze semblent moins bien résister à l'usure, en face du caoutchouc, des polyamides ou des polyesters, que les aciers chromés, nitrurés ou émaillés; toutefois, les expériences en la matière sont trop récentes et on pourra juger des résultats dans quelques années.

5) Les joints métal sur métal, type Pacific, donnent dans certaines régions, d'excellents résultats, pour de faibles diamètres, après 40000 heures, lorsqu'ils sont montés avec beaucoup de soin et que les eaux sont peu chargées. Dans d'autres cas, ils donnent lieu à des fuites exagérées après 10000 heures.

6) On peut en dire de même pour les joints caoutchouc type «Linatex» qui ont, maintenant, leur pourcentage normal de détracteurs et de partisans.

7) Joints labyrinthes. - Les joints labyrinthes, ou joints à fuites, semblent avoir la faveur croissante des exploitants; ce sont des joints à fuites sensiblement constantes dans le temps, ce qui fait que leurs détracteurs estiment ces fuites trop grandes à l'origine; leurs partisans considèrent que ces mêmes fuites sont toujours limitées et connues; l'usure est faible.

Joints de pales. - Ceux-ci ne semblent plus poser de graves problèmes.

Joints statiques. - Il s'agit de joints d'assemblages réalisant les étanchéités entre pièces fixes; une seule conclusion générale semble s'imposer : l'intérêt de la généralisation des joints doubles qui présentent, comme avantage, annexe mais fondamental, de permettre, par injection entre les deux joints, une vérification sans démontage.

\section{ASSEMBLAGES BOULONNÉS :}

La fixation de pièces tournantes lourdes, en porte-à-faux horizontal, a nécessité l'utilisation d'assemblages par goujons et écrous de gros diamètres (70 à $100 \mathrm{~mm}$ ) et de faibles longueurs relatives.

Les goujons étant soumis à des contraintes cycliques de plusieurs hbars lors de la rotation du moulinet, il est nécessaire d'assurer une importante contrainte de serrage qu'il n'est pas simple de déterminer par les procédés classiques.
Les écrous sont, en effet, serrés en faisant subir au goujon un allongement, soit par canne chauffante introduite dans un trou pratiqué dans le goujon, soit par vérins, en tournant ensuite l'écrou à la main jusqu'à ce qu'il repose sur la face d'appui et en laissant le goujon reprendre sa longueur initiale.

Les difficultés sont de deux ordres:

a) Déterminer la loi allongement-contrainte de serrage. La solution, qui semble à l'heure actuelle la meilleure, consiste à déterminer cette loi par mise en place de jauges de contraintes sur un goujon placé dans les conditions normales d'utilisation et en appliquant une force de traction à l'aide de vérins. La mesure des allongements, et des rotations correspondantes des écrous, permet de définir alors une technologie assez simple de premier serrage.

b) Obtenir un allongement suffisant pour aboutir à des contraintes de 15 à 25 hbars. La technique des vérins est actuellement la meilleure, mais il semble que l'utilisation judicieuse de cannes chauffantes de puissances suffisantes puisse donner de bons résultats.

Les quelques incidents constatés étaient dus à une insuffisance des contraintes de serrage, vraisemblablement liée à l'insuffisance du serrage initial, mais aussi à un matage progressif des filets qui nécessite un nouveau serrage à la première visite.

Les essais en cours, sur la technologie de ces types d'assemblages, devraient permettre d'aboutir à une excellente fiabilité dans un avenir très proche.

Outre les trois problèmes les plus importants qui précèdent, on peut signaler quelques incidents ou inconvénients mineurs.

- Le BRUIT: Les groupes bulbes sont un peu plus bruyants que les groupes classiques.

- Cercle de vannage: Lors de la fermeture du vannage, les ruptures de biellettes ou de goujons de cisaillement du vannage semblent un peu plus fréquentes sur les groupes bulbes que pour les Kaplan, vraisemblablement parce que les corps entre deux eaux, qui franchissent les directrices sont plus nombreux en raison du type d'écoulement à faible profondeur, et de l'espacement généralement grand des grilles de prise d'eau $(150$ à $200 \mathrm{~mm})$. Peut-être le phénomène est-il seulement plus sensible aux exploitants de groupes bulbes car le nombre de groupes par usine y est généralement plus grand..

- RÉgulation de vitesse : Les résultats d'essais laissent penser que la faible valeur de l'inertie des parties tournantes pose quelques problèmes, mais ceux-ci ne sont pas sensibles en exploitation courante.

Dans certains cas (fig. 6 a), le remplacement de la loi linéaire de fermeture du vannage par une loi fermeturetemps, composée de deux droites, a pu apporter des améliorations notables pour les surpressions, les dépressions et les mouvements d'arbre, comme le montre la figure $6 \mathrm{~b}$. Ces améliorations ont pu, en particulier, être mises en évidence par la C.N.R. par suite des mesures systématiques faites lors de la mise en service des groupes et comportant l'enregistrement, sur une même bande, des lois de manœuvre du vannage, des pales et de la vanne aval, de la vitesse, des pressions au droit des pales et du distributeur, comme sur 


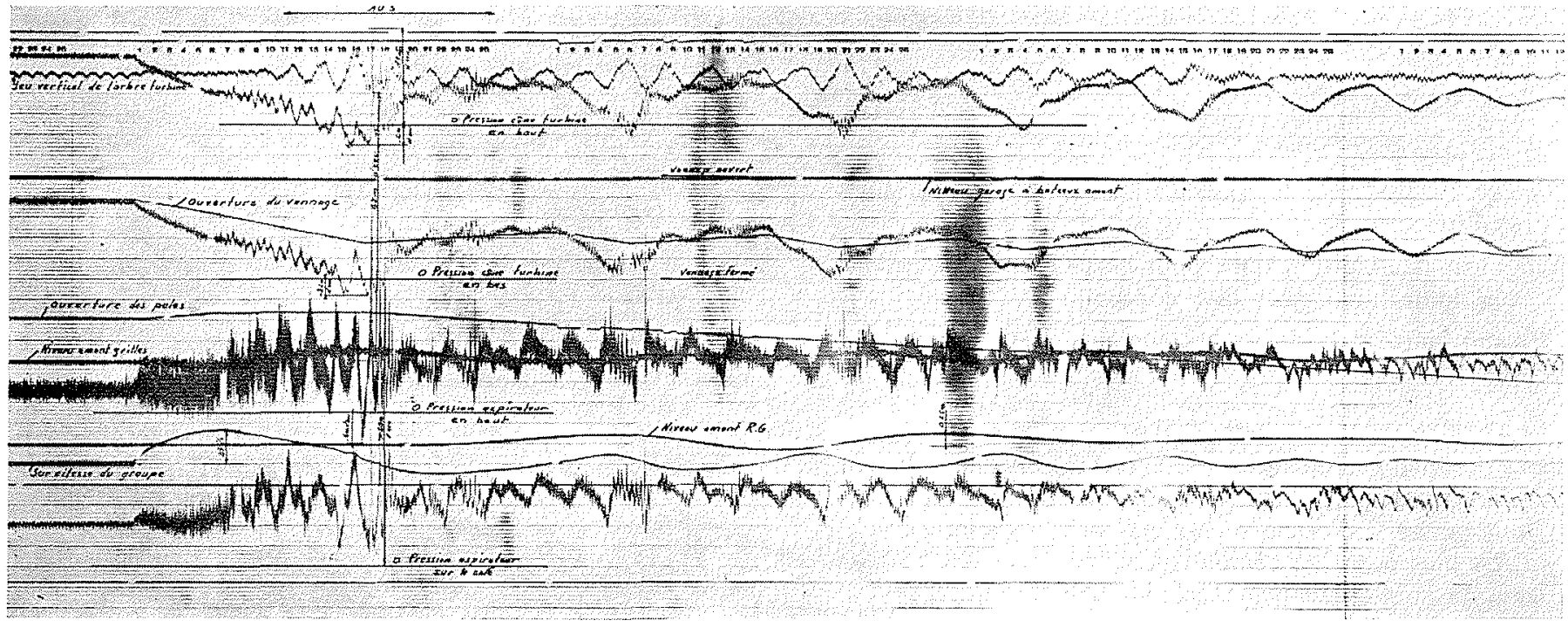

6 a/ Passage en marche à vide, par une loi linéaire de fermeture pales vannage.

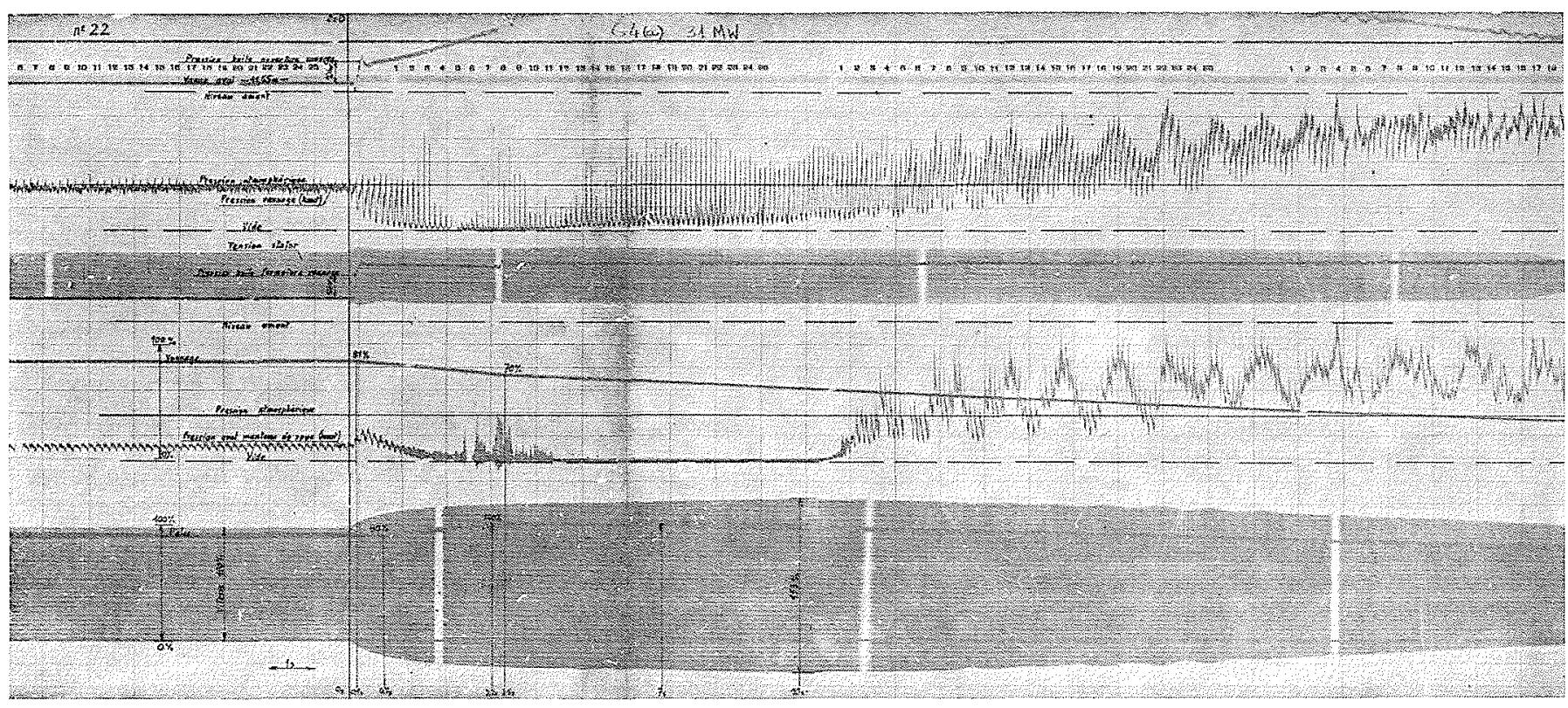

6 b/ Passage en marche à vide, par une loi «cassée» de fermeture pales vannage.

le cône turbine. Les déplacements de l'arbre au droit du palier de la roue sont également mesurés dans les plans horizontaux et verticaux. Toutes ces mesures sont faites aussi bien en régime transitoire qu'en régime établi.

- Emballements. - Marche in déchargeur. - Vannes AVAL: Les marches en déchargeur, les emballements et les fonctionnements par vanne aval semblent ne poser aucun problème majeur avec les groupes bulbes.

- Maintenance. - Aspect Economique: Le faible nombre d'années de comparaison, et le fait de la grande jeunesse des bulbes par rapport aux Kaplan, ne permettent pas de comparaison parfaitement objective des frais d'entretien, qui apparaîtraient comme très légèrement supérieurs pour les bulbes, de façon d'ailleurs peu significative: il est vraisemblable que les indisponibilités, dues aux erreurs de jeunesse, ont grevé aussi lourdement le budget des turbines Kaplan vers les années 1920, mais le nombre élevé d'années de fonctionnement en atténue les effets, aussi bien sur le plan comptable que dans le domaine psychologique. Les indisponibilités des groupes traditionnels et des groupes bulbes sont comparables.

Du point de vue des facilités d'entretien et de maintenance, quelques grandes idées générales sont communes à tous les exploitants des groupes bulbes. 
10 Pour les petits bulbes:

Tout dépend de la conception de la manutention des groupes : celle-ci est excellente dans les dernières réalisations.

$2^{\circ}$ Pour les gros bulbes:

a) ALTERNATEUR :

Contre: l'exiguïté de la machine qui complique certaines réparations.

Pour : leur relative simplicité, pour l'excitation par exemple, sans doute aussi pour leur conception moderne.

En moyenne: prix de revient comparable entre bulbes et groupes classiques.

b) TURBINE :

Pour: les petites réparations, les rechargements éventuels sont plus simples (position horizontale des organes), surtout quand l'accès ne se fait pas par l'alternateur. Il est plus facile de démonter un demi-manteau de roue que de monter un plancher sous une roue Kaplan.

Contre: Le démontage-remontage d'un groupe bulbe est en général plus délicat et plus long que celui d'un groupe vertical. (On peut espérer que ces démontages seront exceptionnels.)

\section{Conclusion}

L'économie réalisée lors de la construction n'augmente pas les indisponibilités ou les difficultés d'exploitation.

\section{Bibliographie}

[1] Liste bibliographique d'études récentes concernant les groupes bulbes. E.D.F., Direction des Etudes et Recherches, Centre de Documentation. Bibl. $N^{\circ} 316,25$ juin 1964,14 p., 84 articles cités.

[2] P. Campmas. - Analyse du fonctionnement d'un groupe bulbe grâce à des prospections de l'écoulement en amont et en aval de la roue. $L a$ Houille Blanche, $n^{\circ} 3$, mai-juin 1961.

[3] MM. Boussuges, Campmas, Cazenaye, Chevalyer, Lecrique. - La régulation de vitesse des groupes bulbes de fortes puissances. - S.H.F., 9० Journées de l'Hydraulique, 1966 , $15 \mathrm{p}$.

[4] P. Cazenave. - Un exemple d'aménagement de basse chute équipéc de groupes bulbes: Pierre-Bénite sur le Rhône. R.G.E., 1964.

[5] Etudes récentes: Fonctionnement en déchargeur des turbines hélices à pales orientables. Session S.H.F. des 16 et 17 novembre 1967. La Houlle Blanche, $\mathrm{n}^{\circ} 2-3 / 1968$.

\section{Discussion}

Président: M. P. Cazenave

M. le Président remercie vivement M. ANDRÉ pour son exposé très synthétique et particulièrement bien ordonné. Il ouvre la discussion.

M. R. LANGLors souhaiterait obtenir quelques renseignements sur les résultats d'exploitation des groupes de basse chute un peu spéciaux, installés à l'étranger notamment par la Société R.W.E. sur la Moselle. Dans ce dernier cas, il s'agit de groupes bulbe d'une puissance de l'ordre de 4 à $6 \mathrm{MW}$ qui ont la particularité d'être munis de multiplicateurs de vitesse co-axiaux du type planétaire qui portent la vitesse de rotation des alternateurs à $750 \mathrm{tr} / \mathrm{mn}$.

M. ANDRÉ communique les informations suivantes qu'il a recueillies auprès des exploitants des usines de la Moselle, visées par M. LANGLoIs. Les plus anciens de ces groupes totalisent onze années de fonctionnement. En ce qui concerne les multiplicateurs de vitesse, les quelques incidents survenus au début de leur mise en service étaient liés, soit aux matériaux employés initialement, soit à des défauts dans les aciers, soit à la qualité de l'huile utilisée. Depuis que ces premiers incidents ont été palliés, il n'y a eu, ni usure, ni incident notable sur ces multiplicateurs de vitesse. Les seuls désagréments constatés sur les groupes de la Moselle concernent la cavitation sur le manteau. Le manteau était en acier ordinaire; on a dû le changer sur $70 \mathrm{~cm}$ dans le plan moyen des pales pour le remplacer par un manteau en acier inoxydable. Depuis que cette modification a été faite, il n'y a plus eu d'ennuis.

M. Pestre (Production Hydraulique E.D.F.) apporte d'intéressantes informations sur la «démontabilité » des groupes bulbe.

La substitution des groupes bulbe aux groupes Kaplan, classiques, dit-il, a suscité à l'origine une certaine inquiétude de la part des exploitants qui redoutaient un accroissement des difficultés de démontabilité et d'accessibilité des organes sur lesquels des interventions d'entretien sont nécessaires.

L'expérience a montré qưil n'en était rien. En effet, les manteaux (démontables) et les pales, qui nécessitent les plus grosses interventions pour recharge des usures par abrasion ou cavitation, s'avèrent bien plus accessibles que dans les groupes Kaplan verticaux.

De même les incidents survenus sur les pôles des alternateurs de Pierre-Bénite ont montré que les temps de démontage de ceux-ci pour remplacement des barres d'amortisseurs détériorées par «étincelage» se sont avérés beaucoup plus courts que ceux que l'on craignait et du même ordre que ceux afférents aux groupes verticaux.

Par contre, le joint charbon, le palier-butée et les enroulements statoriques sont difficilement accessibles; mais fort heureusement les interventions nécessitées par ces organes, le joint charbon mis à part, deviennent plus rares.

Au total, le bilan est plutôt positif pour l'exploitant,

A titre d'exemple, sur le groupe bulbe de Beaumont-Monteux, la rupture d'un tube d'alimentation du servo-moteur des pales dont le démontage eût nécessité plusieurs semaines dans un groupe Kaplan classique a pu être réalisé en moins d'une semaine.

M. le Président remercie M. Pestre et précise qu'à Pierre-Bénite, les soixante-douze pôles des quatre groupes ont été remplacées par d'autres en un mois et demi, et qu'en général, un même alternateur n'a pas été immobilisé plus de deux semaines.

Les sujets traités par M. André, observe M. CASACCI, collvrent un champ tellement vaste qu'il faudrait plusieurs heures pour répondre à toutes les questions qu'ils soulèvent.

Je voudrais seulement évoquer le probleme des joints toumants. Le bon fonctionnement de ces organes dépend de tant de phénomènes ressortissant à des disciplines différentes qu'il n'est pas étonnant que le pourcentage des incidents reste élevé. Je rappelle que les constructeurs de la Rance proposèrent d'entreprendre, avant la réalisation de ces machines, une étude approfondie des problèmes posés par les joints tournants. Ils montrèrent lintérêt de construire une installation expérimentale permettant d'essayer les joints de grands diamètres. Ces propositions restèrent lettre morte. Jignore encore les actions propitiatoires qui assurent le bon fonctionnement des joints des machines de la Rance et ne puis que remercier la déesse Fortune 
d'avoir bien voulu éviter l'infortune du client et des constructeurs. Malgré les efforts dispersés des constructeurs jusqu'à ce jour, le bon fonctionnement des joints tournants reste toujours aléatoire. Pour rendre ces organes entièrement fiables, il faudrait consentir des investissements d'une telle ampleur qu'aucun constructeur ne pourrait l'envisager. Souhaitons qu'un jour des organismes d'Etat, des clients avisés, prennent conscience de l'importance à l'échelle nationale des pertes qu'occasionnent dans tous les domaines industriels, les incidents de fonctionnement des joints tournants et qu'ils se décident à entreprendre des études approfondies sur ces organes.

\section{Abstract}

\section{From Cambeyrac to Strasbourg: fifteen years of bulb turbine operation}

Tests on model and prototype bulb sets have shown their hydraulic performance to be roughly the same or slightly better than that of conventional Kaplan sets. Electrical losses are a little higher in bulb generators, but often with a lower temperature rise.

After fifteen years of operating experience, bulb sets can be claimed to answer the purpose for which they were developed. For example :-

1. Small bulb sets provide the only answer for the heads and discharges associated with very low-output applications.

2. The larger bulb sets have got over their 'teething troubles', which were mainly connected with electro-erosion, loss of insulation efficiency owing to pollution, sealing problems, bolted assemblies, and cavitation. Though these problems have also been experienced with other designs, they were sometimes more crucial on bulb sets.

Bulb and Kaplan running costs appear to be comparable, but this can only be taken as a tentative estimate in view of the limited experience available with bulb sets compared to Kaplans; when the first bulb sets appeared, Kaplans had been operational for 40 years. It would appear, however, that the saving on investment cost associated with bulb units is not achicved at the plant operator's expense. 
